\title{
Intrinsic Tongue Muscle Transverse Component
}

National Cancer Institute

\section{Source}

National Cancer Institute. Intrinsic Tongue Muscle Transverse Component. NCI

Thesaurus. Code C52706.

The intrinsic muscles spanning the length of the tongue that move the tip of the tonge up and down. 